\title{
Quantum codes based on fast pauli block transforms in the finite field
}

\author{
Ronghua Shi · Ying Guo • Moon Ho Lee
}

Received: 20 July 2009 / Accepted: 25 February 2010 / Published online: 10 March 2010

C The Author(s) 2010. This article is published with open access at Springerlink.com

\begin{abstract}
Motivated by the fast Pauli block transforms (or matrices) over the finite field $G F(q)$ for an arbitrary number $q$, we suggest how to construct the simplified quantum code on the basis of quadratic residues. The present quantum code, which is the stabilizer quantum code, can be fast generated from an Abelian group with commutative quantum operators being selected from a suitable Pauli block matrix. This construction does not require the dual-containing or self-orthogonal constraint for the standard quantum error-correction code, thus allowing us to construct a quantum code with much efficiency.
\end{abstract}

Keywords Pauli block matrix - Quadratic residues · Abelian group · Quantum error correction code

\section{Introduction}

The Legendre symbol have already been applied in signal processing, image compression, communications and information, etc. [1,2]. Since the Legendre symbol can be calculated easily, it plays an important role in encoding, cryptography, and so on. However, it is difficult to enrich the applications of the Legendre symbol due to the complexity of the construction processing in terms

R. Shi · Y. Guo $(\bowtie)$

School of Information Science \& Engineering, Central South University, 410083 Changsha, China

e-mail: sdguoying@gmail.com

Y. Guo

Department of Physics, Tsinghua University, 10084 Beijing, China

M. H. Lee

Institute of Information \& Communication, Department of Information \& Communication

Engineering, Chonbuk National University, 561-756 Chonju, Korea 
of its characteristics. Thus, it is a challenge to design an efficient approach to search for the application of the Legendre symbol in terms of matrices or transforms.

Provided an arbitrary finite field $G F(q)$, Euler's criterion $\mathcal{L}_{q}(x)$ for the Legendre symbol is defined by $\mathcal{L}_{q}(x)=x^{\frac{q-1}{2}} \bmod q$, where $q$ is a power of an odd prime number. Namely, $\mathcal{L}_{q}(0)=0, \mathcal{L}_{q}(x)=1$ if $x=y^{2}$ for some element $y \in G F(q)$, and $\mathcal{L}_{q}(x)=-1$ if $x \neq y^{2}$ for any element $x \in G F(q)$. Based on quadratic residues in $G F(q)$, one obtains a matrix $Q_{q}=\left(a_{i j}\right)_{q \times q}$ with elements $a_{i j}=\mathcal{L}_{q}(i-j)$. Taking any two rows of $Q_{q}$, i.e., $\vec{a}_{i}=\left(\mathcal{L}_{q}\left(x_{0}\right), \mathcal{L}_{q}\left(x_{1}\right), \ldots, \mathcal{L}_{q}\left(x_{q-1}\right)\right), \vec{a}_{i+s}=\left(\mathcal{L}_{q}\left(x_{0}+\right.\right.$ $\left.s), \mathcal{L}_{q}\left(x_{1}+s\right), \ldots, \mathcal{L}_{q}\left(x_{q-1}+s\right)\right)$ for $s \neq 0$, it is easy to prove that $\vec{a}_{i} \cdot \vec{a}_{i+s}=$ $\sum_{i=0}^{q-1} \mathcal{L}_{q}\left(x_{i}\right) \mathcal{L}_{q}\left(x_{i}+s\right)=q-1 \bmod q$. This property can be elegantly used for quantum signal processing [3].

Recently, there is a striking development in the stabilizer quantum error-correction code (QECC), whose problem has been reduced to that of searching for the classical dual-containing codes [4-14]. The virtue of this approach is that this code can be directly constructed from the classical codes with certain property, rather than developing a new theory of QECC from scratch. Unfortunately, the need for dualcontaining codes presents a substantial obstacle to the quantum coding theory in its entirety, especially in the context of modern codes, such as convolutional quantum codes and low-density parity-check quantum codes [13]. To resolve this problem, we consider the construction of codes over the finite field $G F(q)$ by employing matrix $Q_{n}$ based on the Legendre symbol. Namely, we first replace all entries of $Q_{n}$ with some Pauli matrices [3], and hence obtain the Pauli block matrix denoted by $\mathcal{Q}_{n}$. After that, we extend the yielded block matrices for the constructions of large-size Pauli block matrices by using the recursive techniques [15-20], along with the Paley matrices because of its smart constructions with Hadmard transforms and high order Hadamard matrices [21]. Since all row-operations that are obtained from any rows of $\mathcal{Q}_{n}$ are independent and commutative, an Abelian group can be elegantly fast generated with efficiency, which may be used for combating against quantum noise or loss in the multi-layer systems of the fractional quantum Hall effect [22]. Due to its beautiful structure, a novel quantum code is fast generated via the stabilizer formalism. This approach provides the great flexibility in designing quantum codes with large code length, and hence allows for an advantage of being simply designed with low complexity.

The structure of the paper is as follows. In Sect. 2, several Pauli block matrices are constructed with fast algorithms. In Sect. 3, according to the smart properties of Pauli block matrices, an Abelian group is generated with much efficiency. In Sect. 4, we investigate the smart construction of the stabilizer quantum codes via the stabilizer formalism. Finally, discussions and conclusions are proposed in Sect. 5.

\section{Pauli block matrices}

\subsection{Pauli block matrices}

Pauli matrices are defined by $\mathcal{P}=\left\{\sigma_{j}: 0 \leq j \leq 3\right\}$, where 


$$
\begin{aligned}
& \sigma_{0}=\left(\begin{array}{ll}
1 & 0 \\
0 & 1
\end{array}\right), \quad \sigma_{1}=\left(\begin{array}{ll}
0 & 1 \\
1 & 0
\end{array}\right) \\
& \sigma_{2}=\left(\begin{array}{cc}
0 & -i \\
i & 0
\end{array}\right), \quad \sigma_{3}=\left(\begin{array}{cc}
1 & 0 \\
0 & -1
\end{array}\right),
\end{aligned}
$$

where $i=\sqrt{-1}$. For simplicity, we denote the $2 \times 2$ identity matrix $\sigma_{0}$ by $\mathcal{I}$ in this paper.

Definition 2.1 Denote $\mathcal{Q}=\left([a]_{i j}\right)_{k \times t}$, then $\mathcal{Q}$ is a Pauli block matrix if and only if all entries $[a]_{i j}$ belong to $\mathcal{P}$, i.e., $[a]_{i j} \in \mathcal{P}$.

Based on the defined matrix $Q_{q}$ with respect to quadratic residues in $G F(q)$, we propose an approach for constructing Pauli block matrices with entries $\sigma_{i}, \sigma_{j} \in \mathcal{P} \backslash\left\{\sigma_{0}\right\}$. In a simple way, it follows two Pauli block matrices, i.e.,

1) $\mathcal{Q}_{q}^{(1)}$, which is constructed by replacing ' 0,1 ' in $Q_{q}$ with $\sigma_{i}$, and ' -1 ' with $\sigma_{j}$.

2) $\mathcal{Q}_{q}^{(2)}$, which is constructed by replacing ' 1 ' in $Q_{q}$ with $\sigma_{i}$ and ' $-1,0$ ' with $\sigma_{j}$.

If $q=4 m+3$ for any positive integer $m$, then the $(q+1) \times(q+1)$ matrix can be constructed as

$$
Q_{q+1}=I_{q+1}+\left(\begin{array}{cc}
0 & t^{T} \\
-t & Q_{q}
\end{array}\right)
$$

where $t$ denotes the all- 1 column vector of the length $q$ and $I_{q+1}$ is the $(q+1) \times(q+1)$ identity matrix. As a result, there are two types of Pauli block matrices:

1) $\mathcal{Q}_{q+1}^{(1)}$, which is constructed by replacing ' 0,1 ' in $Q_{q+1}$ with $\sigma_{i}$, and ' -1 ' with $\sigma_{j}$.

2) $\mathcal{Q}_{q+1}^{(2)}$, which is constructed by replacing ' 1 ' in $Q_{q+1}$ with $\sigma_{i}$, and ' $-1,0$ ' with If $q=4 m+1$ for any positive integer $m$, then one constructs the $2(q+1) \times 2(q+1)$ matrix $Q_{2(q+1)}$ by replacing '0' in the matrix

$$
\left(\begin{array}{ll}
0 & t^{T} \\
t & Q_{q}
\end{array}\right)
$$

with the block matrix $R_{0}$ and ' \pm 1 ' with the matrix $R_{ \pm 1}$, where

$$
R_{0}=\left(\begin{array}{cc}
1 & -1 \\
-1 & -1
\end{array}\right), R_{ \pm 1}= \pm\left(\begin{array}{cc}
1 & 1 \\
1 & -1
\end{array}\right)
$$

Then, there is a Pauli block matrices, i.e., $\mathcal{Q}_{2(q+1)}$, which is constructed by replacing ' 1 ' in $Q_{2(q+1)}$ with $\sigma_{i}$, and ' -1 ' with $\sigma_{j}$. 
Example 2.1 We consider $G F(3)=\{0,1,2\}$ with the non-zero squares $1^{2}=1$ $\bmod 3$ and $2^{2}=1 \bmod 3$, and hence $\mathcal{L}_{3}(0)=0, \mathcal{L}_{3}(1)=1$, and $\mathcal{L}_{3}(2)=-1$. With the rows and columns of a matrix $Q_{3}$ being indexed by $\{0,1,2\}$, one obtains

$$
\begin{aligned}
Q_{3} & =\left(\begin{array}{ccc}
0 & 1 & -1 \\
-1 & 0 & 1 \\
1 & -1 & 0
\end{array}\right), \\
Q_{4} & =\left(\begin{array}{cccc}
1 & 1 & 1 & 1 \\
-1 & 1 & 1 & -1 \\
-1 & -1 & 1 & 1 \\
-1 & 1 & -1 & 1
\end{array}\right) .
\end{aligned}
$$

Consequently, one achieves block matrices as follows

$$
\begin{aligned}
\mathcal{Q}_{3}^{(1)} & =\left(\begin{array}{lll}
\sigma_{i} & \sigma_{i} & \sigma_{j} \\
\sigma_{j} & \sigma_{i} & \sigma_{i} \\
\sigma_{i} & \sigma_{j} & \sigma_{i}
\end{array}\right), \\
\mathcal{Q}_{4}^{(1)} & =\left(\begin{array}{llll}
\sigma_{i} & \sigma_{i} & \sigma_{i} & \sigma_{i} \\
\sigma_{j} & \sigma_{i} & \sigma_{i} & \sigma_{j} \\
\sigma_{j} & \sigma_{j} & \sigma_{i} & \sigma_{i} \\
\sigma_{j} & \sigma_{i} & \sigma_{j} & \sigma_{i}
\end{array}\right) .
\end{aligned}
$$

Example 2.2 According to $G F(5)$ with $\mathcal{L}_{5}(1)=\mathcal{L}_{5}(4)=1 \bmod 5$ and $\mathcal{L}_{5}(2)=$ $\mathcal{L}_{5}(3)=-1 \bmod 5$, if the rows and columns of the matrix $Q_{5}$ are indexed by $G F(5)$, one gets two matrices

$$
Q_{5}=\left(\begin{array}{ccccc}
0 & 1 & -1 & -1 & 1 \\
1 & 0 & 1 & -1 & -1 \\
-1 & 1 & 0 & 1 & -1 \\
-1 & -1 & 1 & 0 & 1 \\
1 & -1 & -1 & 1 & 0
\end{array}\right)
$$

and

$$
Q_{12}=\left(\begin{array}{cccccc}
R_{0} & R_{+1} & R_{+1} & R_{+1} & R_{+1} & R_{+1} \\
R_{+1} & R_{0} & R_{+1} & R_{-1} & R_{-1} & R_{+1} \\
R_{+1} & R_{+1} & R_{0} & R_{+1} & R_{-1} & R_{-1} \\
R_{+1} & R_{-1} & R_{+1} & R_{0} & R_{+1} & R_{-1} \\
R_{+1} & R_{-1} & R_{-1} & R_{+1} & R_{0} & R_{+1} \\
R_{+1} & R_{+1} & R_{-1} & R_{-1} & R_{+1} & R_{0}
\end{array}\right)
$$


As a result, one obtains the Pauli block matrices as follows

$$
\mathcal{Q}_{5}^{(1)}=\left(\begin{array}{ccccc}
\sigma_{i} & \sigma_{i} & \sigma_{j} & \sigma_{j} & \sigma_{i} \\
\sigma_{i} & \sigma_{i} & \sigma_{i} & \sigma_{j} & \sigma_{j} \\
\sigma_{j} & \sigma_{i} & \sigma_{i} & \sigma_{i} & \sigma_{j} \\
\sigma_{j} & \sigma_{j} & \sigma_{i} & \sigma_{i} & \sigma_{i} \\
\sigma_{i} & \sigma_{j} & \sigma_{j} & \sigma_{i} & \sigma_{i}
\end{array}\right)
$$

and hence

$$
\mathcal{Q}_{12}=\left(\begin{array}{cccccc}
\mathcal{R}_{0} & \mathcal{R}_{+1} & \mathcal{R}_{+1} & \mathcal{R}_{+1} & \mathcal{R}_{+1} & \mathcal{R}_{+1} \\
\mathcal{R}_{+1} & \mathcal{R}_{0} & \mathcal{R}_{+1} & \mathcal{R}_{-1} & \mathcal{R}_{-1} & \mathcal{R}_{+1} \\
\mathcal{R}_{+1} & \mathcal{R}_{+1} & \mathcal{R}_{0} & \mathcal{R}_{+1} & \mathcal{R}_{-1} & \mathcal{R}_{-1} \\
\mathcal{R}_{+1} & \mathcal{R}_{-1} & \mathcal{R}_{+1} & \mathcal{R}_{0} & \mathcal{R}_{+1} & \mathcal{R}_{-1} \\
\mathcal{R}_{+1} & \mathcal{R}_{-1} & \mathcal{R}_{-1} & \mathcal{R}_{+1} & \mathcal{R}_{0} & \mathcal{R}_{+1} \\
\mathcal{R}_{+1} & \mathcal{R}_{+1} & \mathcal{R}_{-1} & \mathcal{R}_{-1} & \mathcal{R}_{+1} & \mathcal{R}_{0}
\end{array}\right)
$$

where $\mathcal{R}_{ \pm 1}$ and $\mathcal{R}_{0}$ are all 2-order Pauli block matrices defined respectively by

$$
\mathcal{R}_{+1}=\left(\begin{array}{cc}
\sigma_{i} & \sigma_{i} \\
\sigma_{i} & \sigma_{j}
\end{array}\right), \quad \mathcal{R}_{0}=\mathcal{R}_{-1}=\left(\begin{array}{cc}
\sigma_{i} & \sigma_{j} \\
\sigma_{j} & \sigma_{j}
\end{array}\right)
$$

Example 2.3 We consider $G F(7)=\{0,1,2,3,4,5,6\}$ with $\mathcal{L}_{7}(1)=\mathcal{L}_{7}(2)=$ $\mathcal{L}_{7}(4)=1$ and $\mathcal{L}_{7}(3)=\mathcal{L}_{7}(5)=\mathcal{L}_{7}(6)=-1$ because of $1^{2}=6^{2}=1$ $\bmod 7,3^{2}=4^{2}=2 \bmod 7$, and $2^{2}=5^{2}=4 \bmod 7$. When the rows and columns of the matrix are indexed by $G F(7)$, we obtain two corresponding matrices as follows

$$
Q_{7}=\left(\begin{array}{ccccccc}
0 & 1 & 1 & -1 & 1 & -1 & -1 \\
-1 & 0 & 1 & 1 & -1 & 1 & -1 \\
-1 & -1 & 0 & 1 & 1 & -1 & 1 \\
1 & -1 & -1 & 0 & 1 & 1 & -1 \\
-1 & 1 & -1 & -1 & 0 & 1 & 1 \\
1 & -1 & 1 & -1 & -1 & 0 & 1 \\
1 & 1 & -1 & 1 & -1 & -1 & 0
\end{array}\right)
$$

and

$$
Q_{8}=\left(\begin{array}{cccccccc}
1 & 1 & 1 & 1 & 1 & 1 & 1 & 1 \\
-1 & 1 & 1 & 1 & -1 & 1 & -1 & -1 \\
-1 & -1 & 1 & 1 & 1 & -1 & 1 & -1 \\
-1 & -1 & -1 & 1 & 1 & 1 & -1 & 1 \\
-1 & 1 & -1 & -1 & 1 & 1 & 1 & -1 \\
-1 & -1 & 1 & -1 & -1 & 1 & 1 & 1 \\
-1 & 1 & -1 & 1 & -1 & -1 & 1 & 1 \\
-1 & 1 & 1 & -1 & 1 & -1 & -1 & 1
\end{array}\right)
$$


Similarly, one obtains the two Pauli block matrices

$$
\mathcal{Q}_{7}^{(1)}=\left(\begin{array}{ccccccc}
\sigma_{i} & \sigma_{i} & \sigma_{i} & \sigma_{j} & \sigma_{i} & \sigma_{j} & \sigma_{j} \\
\sigma_{j} & \sigma_{i} & \sigma_{i} & \sigma_{i} & \sigma_{j} & \sigma_{i} & \sigma_{j} \\
\sigma_{j} & \sigma_{j} & \sigma_{i} & \sigma_{i} & \sigma_{i} & \sigma_{j} & \sigma_{i} \\
\sigma_{i} & \sigma_{j} & \sigma_{j} & \sigma_{i} & \sigma_{i} & \sigma_{i} & \sigma_{j} \\
\sigma_{j} & \sigma_{i} & \sigma_{j} & \sigma_{j} & \sigma_{i} & \sigma_{i} & \sigma_{i} \\
\sigma_{i} & \sigma_{j} & \sigma_{i} & \sigma_{j} & \sigma_{j} & \sigma_{i} & \sigma_{i} \\
\sigma_{i} & \sigma_{i} & \sigma_{j} & \sigma_{i} & \sigma_{j} & \sigma_{j} & \sigma_{i}
\end{array}\right),
$$

and

$$
\mathcal{Q}_{8}^{(1)}=\left(\begin{array}{cccccccc}
\sigma_{i} & \sigma_{i} & \sigma_{i} & \sigma_{i} & \sigma_{i} & \sigma_{i} & \sigma_{i} & \sigma_{i} \\
\sigma_{j} & \sigma_{i} & \sigma_{i} & \sigma_{i} & \sigma_{j} & \sigma_{i} & \sigma_{j} & \sigma_{j} \\
\sigma_{j} & \sigma_{j} & \sigma_{i} & \sigma_{i} & \sigma_{i} & \sigma_{j} & \sigma_{i} & \sigma_{j} \\
\sigma_{j} & \sigma_{j} & \sigma_{j} & \sigma_{i} & \sigma_{i} & \sigma_{i} & \sigma_{j} & \sigma_{i} \\
\sigma_{j} & \sigma_{i} & \sigma_{j} & \sigma_{j} & \sigma_{i} & \sigma_{i} & \sigma_{i} & \sigma_{j} \\
\sigma_{j} & \sigma_{j} & \sigma_{i} & \sigma_{j} & \sigma_{j} & \sigma_{i} & \sigma_{i} & \sigma_{i} \\
\sigma_{j} & \sigma_{i} & \sigma_{j} & \sigma_{i} & \sigma_{j} & \sigma_{j} & \sigma_{i} & \sigma_{i} \\
\sigma_{j} & \sigma_{i} & \sigma_{i} & \sigma_{j} & \sigma_{i} & \sigma_{j} & \sigma_{j} & \sigma_{i}
\end{array}\right)
$$

\subsection{Fast constructions of pauli block matrices}

To construct the large-order Pauli block matrices, we should introduce the Kronecker product of two matrices $A=\left(a_{i j}\right)_{r \times l}$ and $B=\left(b_{i j}\right)_{s \times t}$, i.e.,

$$
A \otimes B=\left(\begin{array}{cccc}
a_{11} B & a_{12} B & \cdots & a_{1 l} B \\
a_{21} B & a_{22} B & \cdots & a_{2 l} B \\
\cdots & \cdots & \cdots & \cdots \\
a_{r 1} B & a_{r 2} B & \cdots & a_{r l} B
\end{array}\right)
$$

With a little abuse, we denote the Kronecker product by using the notation ' $\otimes$ ' throughout this paper.

Lemma 2.1 According to the Kronecker product of any two block matrices $A$ and $B$, one has the following properties

$$
\begin{aligned}
& (A \otimes B)^{T}=A^{T} \otimes B^{T}, \\
& (A \otimes B) \cdot(C \otimes D)=(A \cdot C) \otimes(B \cdot D), \\
& (A \otimes B) \otimes C=A \otimes(B \otimes C), \\
& (A+B) \otimes C=A \otimes C+B \otimes C, \\
& A \otimes(B+C)=A \otimes B+A \otimes C .
\end{aligned}
$$


Lemma 2.2 Let $C$ be an $m \times m$ symmetric Conference matrix [21]. For two $n \times n$ Hadamard matrices $A$ and $B$, the matrix

$$
R=A \otimes C+B \otimes I
$$

is Hadamard matrix, if and only if

$$
A \cdot B^{T}+B \cdot A^{T}=0 .
$$

In particular, all entries of $A$ and $B$ satisfy $a_{i i}=-b_{i i}$ and $a_{i j}=b_{i j}$ for $i \neq j$.

As an example, taking $n=2$, we have

$$
A_{2}=\left(\begin{array}{cc}
-1 & 1 \\
1 & 1
\end{array}\right), \quad B_{2}=\left(\begin{array}{cc}
1 & 1 \\
1 & -1
\end{array}\right)
$$

Proof To check the matrix $R$ is Hadamard, it should satisfy the condition $R \cdot R^{T}=I$. According to Eq. (16), we obtain

$$
R^{T}=A^{T} \otimes C^{T}+B^{T} \otimes I
$$

where $X^{T}$ denotes a transpose of the matrix $X$. From the statement of Paley matrices [21], one has

$$
\begin{aligned}
S \cdot S^{T}= & (A \otimes C+B \otimes I)\left(A^{T} \otimes C^{T}+B^{T} \otimes I\right) \\
= & (A \otimes C)\left(A^{T} \otimes C^{T}\right)+\left(B \cdot B^{T}\right) \otimes I \\
& +\left(A \cdot B^{T}\right) \otimes C+\left(B \cdot A^{T}\right) \otimes C^{T} .
\end{aligned}
$$

Since $C^{T}=C, A^{T}=A$ and $C^{T}=C$, it is evident that the matrices $C, A, B$ and $A \otimes C$ are all orthogonal matrices. Thus, we obtain

$$
R \cdot R^{T}=\left(A \cdot B^{T}+B \cdot A^{T}\right) \otimes C+I .
$$

Namely, we have $R \cdot R^{T}=I$, if and only if

$$
A \cdot B^{T}+B \cdot A^{T}=0
$$

which holds the relations of two matrices $A$ and $B$ for $n=2$ expressed in Eq. (18).

Making use of the above Paley construction approach to obtain the high order Hadamard matrices for our construction, we derive the relationship for two Hadamard matrices $A$ and $B$ that can be useful while constructing the matrix $Q_{2(q+1)}$ in the finite field $G F(q)$, and hence the Pauli block matrix $\mathcal{Q}_{2(q+1)}$.

Definition 2.2 The pair $(A, B)$ of Hadamard matrices is called as the matched matrices if and only if Eq. (22) holds.

Based on above discussion, Paley construction can be summarized as follows. 
1) The pair $(A, B)$ of two Hadamard matrices $A$ and $B$ is matched.

2) The Conference matrix $C$ must be selected properly to ensure the constructed matrix $R$ satisfy the restriction condition, i.e., $R \cdot R^{T}=I$.

If we extend the matched pairs to more couples, the corresponding matrices in terms of Paley construction approaches can be generalized with much efficiency. Now, we investigate the constructions of more matched pairs with two statements.

Proposition 2.1 If the pair $(A, B)$ is matched and $D$ is a Hadamard matrix, then the pair $(A \otimes D, B \otimes D)$ is matched.

Proposition 2.2 If two matrices $X$ and $Y$ are both $n \times 2 n$ matrices and the composite matrix $U$ is the Hadamard matrix, then the pair $(U, V)$ is matched, where

$$
U=\left(\begin{array}{l}
X \\
Y
\end{array}\right), \quad V=\left(\begin{array}{c}
Y \\
-X
\end{array}\right) \text {. }
$$

Making use of the kronecker product of Pauli block matrices [15-20], a family of Pauli block matrices may be extended with the fast algorithm.

Theorem 2.1 Suppose $\mathcal{Q}_{q}$ and $\mathcal{Q}_{p}$ are two Pauli block matrices defined in the respective finite fields $G F(q)$ and $G F(p)$. For any nonnegative integer numbers $s$ and $m$, a large-order block Jacket matrix $\mathcal{Q}_{q^{s}} p^{m}$ may be constructed (or decomposed) in the following way:

$$
\begin{aligned}
\mathcal{Q}_{q^{s} p^{m}}= & \left\{\mathcal{I}_{q^{s}} \otimes\left(\prod_{i=0}^{m-1} \mathcal{I}_{p^{m-i-1}} \otimes \mathcal{Q}_{p} \otimes \mathcal{I}_{p^{i}}\right)\right\} \\
& \times\left\{\left(\prod_{i=0}^{s-1} \mathcal{I}_{q^{s-i-1}} \otimes \mathcal{Q}_{q} \otimes \mathcal{I}_{q^{i}}\right) \otimes \mathcal{I}_{p^{m}}\right\} \\
= & \left\{\mathcal{I}_{q^{s}} \otimes\left(\prod_{i=1}^{m} \mathcal{I}_{p^{m-i}} \otimes \mathcal{Q}_{p} \otimes \mathcal{I}_{p^{i-1}}\right)\right\} \\
& \times\left\{\left(\prod_{i=1}^{s} \mathcal{I}_{q^{s-i}} \otimes \mathcal{Q}_{q} \otimes \mathcal{I}_{q^{i-1}}\right) \otimes \mathcal{I}_{p^{m}}\right\} .
\end{aligned}
$$

Proof Based on an arbitrary Pauli block matrix $\mathcal{Q}_{r}$, the large-order Pauli block matrices $\mathcal{Q}_{r} l$ for $l \geq 2$ can be obtained by using the recursive relations,

$$
\begin{aligned}
\mathcal{Q}_{r l} & =\mathcal{Q}_{r^{l-1}} \otimes \mathcal{Q}_{r} \\
& =\prod_{i=0}^{l-1} \mathcal{I}_{r^{l-i-1}} \otimes \mathcal{Q}_{r} \otimes \mathcal{I}_{r^{i}} \\
& =\prod_{i=1}^{m} \mathcal{I}_{r^{l-i}} \otimes \mathcal{Q}_{r} \otimes \mathcal{I}_{r^{i-1}},
\end{aligned}
$$


Table 1 Comparisons of the computation complexity of several related algorithms for constructing Pauli block matrices

\begin{tabular}{llll}
\hline & Direct approach $\left(\mathcal{Q}_{n}\right)$ & Proposed algorithm $\left(n=p^{m}\right)$ & Proposed algorithm $\left(n=q^{s} p^{m}\right)$ \\
\hline Add. & $(n-1) n$ & $(p-1) n m$ & $(p+q-2) n$ \\
Mul. & $n^{2}$ & $u_{p} n m$ & $u_{p} m n+u_{q} s n$ \\
\hline
\end{tabular}

For simplicity, Add. and Mul. denote the number of additions and multiplications. $u_{p}$ or $u_{q}$ expresses the number of non-identity matrices in Pauli block matrices

where $r \in\{p, q\}$ and $l \in\{s, m\}$. According to the properties of the Kronecker product, it is easy to calculate

$$
\mathcal{Q}_{q^{s} p^{m}}=\mathcal{Q}_{q^{s}} \otimes \mathcal{Q}_{p^{m}}=\left(\mathcal{I}_{q^{s}} \otimes \mathcal{Q}_{p^{m}}\right) \cdot\left(\mathcal{Q}_{q^{s}} \otimes \mathcal{I}_{p^{m}}\right),
$$

and then this completes the proof of the theorem.

According to the above discussions, the large order Pauli block matrices can be constructed with the fast algorithm by employing Pauli Block matrices $\mathcal{Q}_{n}$ in two finite fields $G F(p)$ and $G F(q)$ with respect to the Kronecker product expressed in Eq. (24). The computational complexity of the proposed algorithm is shown in Table 1. As an example, the construction of $\mathcal{Q}_{15}=\mathcal{Q}_{3} \otimes \mathcal{Q}_{5}$ is depicted in Fig. 1. According to Table 1, the computation of the combined Pauli block matrix $\mathcal{Q}_{15}$ requires 26 additions and 34 multiplications. Compared with the direct computation approach, the proposed algorithm is obviously faster.

It is necessary to note that the Pauli block matrix $\mathcal{Q}$ can be fast constructed with fast algorithms. The yielded matrix is important in this paper since it will be elegantly utilized for the constructions of the stabilizer quantum codes in next section. Although it does not seem to have a direct impact on the characteristics of the proposed quantum codes, it proposes an elegant approach to reduce the process of encoding while designing the stabilizer of quantum codes in an Abelian field.

\section{Abelian groups generated from Pauli block matrices}

We denote $\mathcal{P}^{\otimes n}$ the set of $n$-fold tensor products (the Kronecker product) of Pauli operators (matrices) in $\mathcal{P}$. Then $\mathcal{P}^{\otimes n}$, together with possible multiplicative factors in $\{ \pm i, \pm 1\}$, form a group of $n$-qubit operations, denoted by $\mathcal{G}_{n}$. An arbitrary operation $\alpha_{u} \in \mathcal{G}_{n}$ can be uniquely expressed by [3]

$$
\alpha_{u}=i^{\lambda}\left[\sigma_{1}^{x_{u 1}} \sigma_{2}^{z_{u 1}}\right] \otimes \cdots \otimes\left[\sigma_{1}^{x_{u n}} \sigma_{2}^{z_{u n}}\right]
$$

where $x_{u t}, z_{u t} \in\{0,1\}$ for $1 \leq t \leq n$. Omitting factor $i^{\lambda}$, we denote $\alpha_{u}$ by a concatenated $2 n$-dimensional vector $\vec{\alpha}_{u}$ [12],

$$
\vec{\alpha}_{u}=\left(\vec{x}_{u} \mid \vec{z}_{u}\right)=\left(x_{u 1}, \ldots, x_{u n} \mid z_{u 1}, \ldots, z_{u n}\right) .
$$




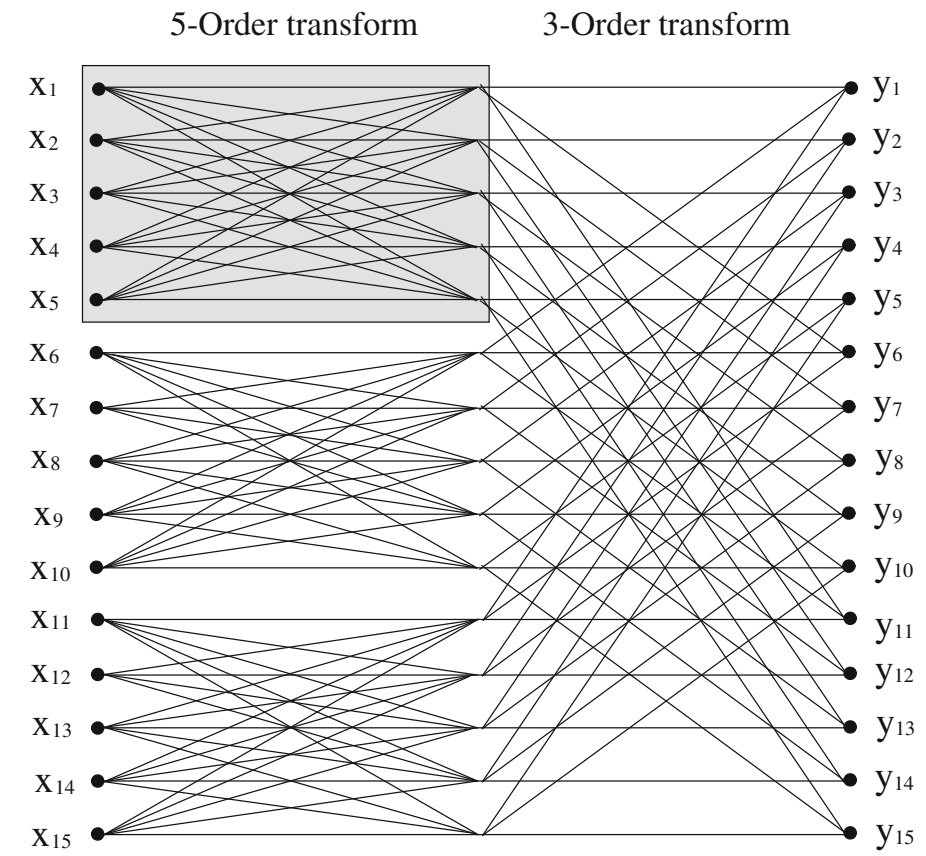

Fig. 1 Signal flow graph of the fast Pauli block transform for matrix $\mathcal{Q}_{15}$.

The Hamming weight of $\vec{\alpha}_{u}$ is the number of $\left(x_{u h} \mid z_{u h}\right)(1 \leq h \leq n)$ such that $\left(x_{u h} \mid z_{u h}\right) \neq(0 \mid 0)$. The symplectic inner product of any two vectors $\vec{\alpha}_{u}=\left(\vec{x}_{u} \mid \vec{z}_{u}\right)$ and $\vec{\alpha}_{v}=\left(\vec{x}_{v} \mid \vec{z}_{v}\right)$ is defined by

$$
\vec{\alpha}_{u} \cdot \vec{\alpha}_{v}=\vec{x}_{u} \cdot \vec{z}_{v}+\vec{z}_{u} \cdot \vec{x}_{v}
$$

where $\vec{x}_{u} \cdot \vec{z}_{v}=\sum_{i=1}^{n} x_{u i} z_{v i}$ and $\vec{z}_{u} \cdot \vec{x}_{v}=\sum_{i=1}^{n} z_{u i} x_{v i}$. According to Ref. [12], two operations $\alpha_{u}$ and $\alpha_{v}$ commute if and only if

$$
\vec{\alpha}_{u} \cdot \vec{\alpha}_{v}=0
$$

The symplectic inner product of two vectors is important since it can be used conveniently to search for generators of an Abelian subgroup $\mathcal{S} \subseteq \mathcal{G}_{n}$.

Assume that each row of a Pauli block matrix $\mathcal{Q}_{n}$ is denoted by $\left(\sigma_{i_{1}}, \ldots, \sigma_{i_{n}}\right)$ for $1 \leq i \leq n$, from which an $n$-qubit operation, called as the row operator, can be obtained as

$$
\alpha_{i}=\sigma_{i_{1}} \otimes \cdots \otimes \sigma_{i_{n}}
$$

Based on properties of the Kronecker product [15,16,19], we achieve the commutativity of row operators for Pauli block matrices $\mathcal{Q}_{n}$. 
Theorem 3.1 For Pauli block matrices $\mathcal{Q}_{n}$ yielded in the finite field, independent row operators constructed from rows of $\mathcal{Q}_{n}$ are all commuting, and hence generate an Abelian group.

Proof According to Pauli block matrices $\mathcal{Q}_{n}$ that are constructed by substituting Pauli matrices for the entries of matrices $Q_{n}$ in previous section, one obtain the $n$-qubit operations from all rows of $\mathcal{Q}_{n}$, which can be expressed by the concatenated vectors in Eq. (26). As a result, an $n \times 2 n$ matrices $\left(H_{x} \mid H_{z}\right)$ can be constructed from these vectors. According to the properties of the Hadamard matrices or the combined matrices $R$ with the pair $(A, B)$, it is easy to calculate

$$
H_{x} \cdot H_{z}^{T}+H_{z} \cdot H_{x}^{T}=0 \bmod 2,
$$

which implies that all independent $n$-qubit row operators of $\mathcal{Q}_{n}$ are commuting [11].

Corollary 3.1 Considering any two given Pauli block matrices $\mathcal{Q}_{p^{m}}$ and $\mathcal{Q}_{q^{s}}$, all independent $\left(q^{s} p^{m}\right)$-qubit row operators of the Kronecker product $\mathcal{Q}_{q^{s}} p^{m}=\mathcal{Q}_{p^{m}} \otimes \mathcal{Q}_{q^{s}}$ are commuting.

Example 3.1 According to the finite field $G F(2)=\{0,1\}$ with the non-zero squares $1^{2}=1 \bmod 2$, we have $\mathcal{L}_{2}(0)=0$ and $\mathcal{L}_{2}(1)=1$. With the rows and columns of a matrix $Q_{2}$ indexed by $\{0,1\}$, one achieves

$$
Q_{2}=\left(\begin{array}{ll}
0 & 1 \\
1 & 0
\end{array}\right)
$$

Then one obtains the Pauli block matrix

$$
\mathcal{Q}_{2}^{(2)}=\left(\begin{array}{ll}
\sigma_{i} & \sigma_{j} \\
\sigma_{j} & \sigma_{i}
\end{array}\right),
$$

which is the smallest sized Pauli block matrix for our construction. Taking $i=1$ and $j=3$, one has the concatenated matrix

$$
H_{2}=\left(H_{2}^{x} \mid H_{2}^{z}\right)=\left(\begin{array}{l|l}
10 & 01 \\
01 & 10
\end{array}\right) \text {. }
$$

It is easy to check that $H_{2}^{x} \cdot H_{2}^{z T}+H_{2}^{z} \cdot H_{2}^{x T}=0 \bmod 2$, which means that two row operators of $\mathcal{Q}_{2}^{(2)}$

$$
\alpha_{1}=\sigma_{1} \otimes \sigma_{3}, \alpha_{2}=\sigma_{3} \otimes \sigma_{1},
$$

are commuting. 
Example 3.2 Taking $i=1$ and $j=3$ for the Pauli block matrix $\mathcal{Q}_{3}^{(1)}$, one has the concatenated matrix

$$
H_{3}=\left(H_{3}^{x} \mid H_{3}^{z}\right)=\left(\begin{array}{l|l|l}
110 & 001 \\
011 & 100 \\
101 & 010
\end{array}\right) \text {. }
$$

It is obvious that $H_{3}^{x} \cdot H_{3}^{z T}+H_{3}^{z} \cdot H_{3}^{x T}=0 \bmod 2$, and hence three row operators of $\mathcal{Q}_{3}^{(1)}$

$$
\begin{aligned}
& \alpha_{1}=\sigma_{1} \otimes \sigma_{1} \otimes \sigma_{3}, \\
& \alpha_{2}=\sigma_{3} \otimes \sigma_{1} \otimes \sigma_{1}, \\
& \alpha_{3}=\sigma_{1} \otimes \sigma_{3} \otimes \sigma_{1},
\end{aligned}
$$

are commuting. In a similar way, from the Pauli block matrix $\mathcal{Q}_{4}^{(1)}$ for $i=1$ and $j=3$, one has the concatenated matrix

$$
H_{4}=\left(\begin{array}{l|l|l}
1111 & 0000 \\
0110 & 1001 \\
0011 & 1100 \\
0101 & 1010
\end{array}\right),
$$

from which it is easy to check that all row operators of $\mathcal{Q}_{4}^{(1)}$ are also commuting. In addition, taking $i=1$ and $j=3$ for $\mathcal{Q}_{5}^{(1)}$, one has

$$
H_{5}=\left(\begin{array}{l|l|l}
11001 & 00110 \\
11100 & 00011 \\
01110 & 10001 \\
00111 & 11000 \\
10011 & 01100
\end{array}\right)
$$

It means that five row operators of $\mathcal{Q}_{5}^{(1)}$ for $i=1$ and $j=3$ are commuting. Furthermore, let $i=1$ and $j=3$ in $\mathcal{Q}_{12}$, and then one obtains the concatenated matrix 


$$
H_{12}=\left(\begin{array}{l|l|l}
101111111111 & 010000000000 \\
001010101010 & 110101010101 \\
111011101011 & 000100010100 \\
100010000010 & 011101111101 \\
111110111010 & 000001000101 \\
101000100000 & 010111011111 \\
111011101110 & 000100010001 \\
100010001000 & 011101110111 \\
111010111011 & 000101000100 \\
100000100010 & 011111011101 \\
111110101110 & 000001010001 \\
101000001000 & 010111110111
\end{array}\right) .
$$

It shows that all row operators of $\mathcal{Q}_{12}^{(1)}$ are commuting.

Example 3.2 Taking $i=1$ and $j=3$ for the combined Pauli block matrix

$$
\begin{aligned}
\mathcal{Q}_{6} & =\mathcal{Q}_{2}^{(2)} \otimes \mathcal{Q}_{3}^{(1)} \\
& =\left(\begin{array}{llllll}
\sigma_{1} & \sigma_{1} & \sigma_{2} & \sigma_{3} & \sigma_{3} & \sigma_{1} \\
\sigma_{2} & \sigma_{1} & \sigma_{1} & \sigma_{1} & \sigma_{3} & \sigma_{3} \\
\sigma_{1} & \sigma_{2} & \sigma_{1} & \sigma_{3} & \sigma_{1} & \sigma_{3} \\
\sigma_{3} & \sigma_{3} & \sigma_{1} & \sigma_{1} & \sigma_{1} & \sigma_{2} \\
\sigma_{1} & \sigma_{3} & \sigma_{3} & \sigma_{2} & \sigma_{1} & \sigma_{1} \\
\sigma_{3} & \sigma_{1} & \sigma_{3} & \sigma_{1} & \sigma_{2} & \sigma_{1}
\end{array}\right),
\end{aligned}
$$

one has the concatenated matrix

$$
H_{6}=\left(H_{6}^{x} \mid H_{6}^{z}\right)=\left(\begin{array}{l|l|l}
111001 & 001110 \\
111100 & 100011 \\
111010 & 010101 \\
001111 & 110001 \\
100111 & 011100 \\
010111 & 101010
\end{array}\right),
$$

which shows that all row operators of $\mathcal{Q}_{6}$ are commuting.

\section{Quantum codes based on Pauli block matrices}

On the basis of the stabilizer formalism, we construct quantum codes $C(\mathcal{S})$ by using Pauli block matrices $\mathcal{Q}_{n}$ with commutative row operators, from which $n-k$ independent row operators can be selected as generators of an Abelian group $\mathcal{S}$.

For an Abelian subgroup $\mathcal{S}$ of $\mathcal{G}_{n}$, the stabilizer quantum codes $C(\mathcal{S})$ is a set of $n$-qubit quantum states $\{|\psi\rangle\}$ associated with $\mathcal{S}$, i.e.,

$$
C(\mathcal{S})=\{|\psi\rangle: M|\psi\rangle=|\psi\rangle, \forall M \in \mathcal{S}\},
$$


which is the subspace fixed by $\mathcal{S}$ (called as the stabilizer). For an $[[n, k, d]]$ stabilizer quantum code, which encodes $k$ logical qubits into $n$ physics qubits, $C(\mathcal{S})$ has dimension $2^{k}$ and $\mathcal{S}$ has $2^{n-k}$ independent operators. The present quantum code can correct $t_{c}=\left\lfloor\frac{d-1}{2}\right\rfloor$ quantum errors and detect $t_{d}=d-1$ quantum errors.

To construct such a quantum code, the sticking point is to search for an Abelian group, the stabilizer $\mathcal{S}$, from which the code $C(\mathcal{S})$ can be structurally generated through Eq. (39).

Theorem 4.1 For the yielded (combined) Pauli block matrix $\mathcal{Q}_{n}$ in the finite field, quantum code $C(\mathcal{S})$ can be generated via the the stabilizer formalism with parameters $[[n, k, d]]$, where the stabilizer $\mathcal{S}$ is a set of $n$-qubit operators selected from $n-k$ independent rows of Pauli block matrix $\mathcal{Q}_{n}$.

Proof Suppose that there are $r(r \geq n-k)$ independent rows of $\mathcal{Q}_{n}$. Then $n-k$ generators of the stabilizer $\mathcal{S}$ can be generated by selecting $n-k$ rows from these $r$ independent rows of $\mathcal{Q}_{n}$ provided $n-k \leq r$. Namely, any $n-k$ operators $\alpha_{i_{1}}, \alpha_{i_{2}}, \ldots, \alpha_{i_{n-k}}$ can be selected to generate an Abelian group

$$
\mathcal{S}=\left\langle\alpha_{i_{1}}, \alpha_{i_{2}}, \ldots, \alpha_{i_{n-k}}\right\rangle
$$

which is a stabilizer in essence. By making use of Eq. (39), an stabilizer quantum code $[[n, k, d]]$ can be generated from $\mathcal{S}$. According to the quantum Singleton bound proposed in Ref. [12], it follows that $k \leq n-2 d+2$. This completes the proof of the theorem.

Example 4.1 We consider the Pauli block matrix $\mathcal{Q}_{5}$ with the concatenated matrix $H_{5}=\left(H_{5}^{x} \mid H_{5}^{x}\right)$ being described in Eq. (35). It is known that all rows of $H_{5}$ are independent. Thus, any $5-k$ rows of $H_{5}$, denoted by $\mathcal{H}_{5}=\left(h_{5}^{x} \mid h_{5}^{z}\right)$, can be selected to generate the stabilizer $\mathcal{S}$ with $5-k$ generators. According to the construction conditions of quantum codes in Ref. [11], we get the generator matrix of quantum codes $\mathcal{G}_{5}=\left(g_{5}^{x} \mid g_{5}^{z}\right)$ satisfying

$$
h_{5}^{x} \cdot g_{5}^{z}+h_{5}^{z} \cdot g_{5}^{x}=0
$$

which can be rewritten as

$$
\mathcal{H}_{5} \cdot R \cdot \mathcal{G}_{5}=0
$$

where $R=\left(\begin{array}{cc}0_{5 \times 5} & I_{5 \times 5} \\ I_{5 \times 5} & 0_{5 \times 5}\end{array}\right)$. To construct such a quantum code, we assume that there exists one unitary matrix $U$ such that

$$
U(h R)=\left(I_{(5-k) \times(5-k)} \mid \Lambda_{(5-k) \times(5+k)}\right) .
$$

According to Eq. (41), the generator matrix $\mathcal{G}_{5}$ is calculated

$$
\mathcal{G}_{5}=\left(\Lambda_{(5-k) \times(5+k)}^{T} \mid I_{(5+k) \times(5+k)}\right),
$$


from which quantum codes can be constructed with the parameters $[[5,0,4]],[[5,1,2]]$, $[[5,2,1]]$, [[5,3,1]], and [[5,4,1]].

Taking the quantum code $[[5,1,2]]$ as an example, we select four rows to generate the matrix

$$
\mathcal{H}_{5}=\left(\begin{array}{l|l|l}
11001 & 00110 \\
11100 & 00011 \\
01110 & 10001 \\
00111 & 11000
\end{array}\right)
$$

From Eq. (41), we get

$$
\mathcal{G}_{5}=\left(\begin{array}{l|l|l}
01101 & 00000 \\
00010 & 10000 \\
11010 & 01000 \\
10110 & 00100 \\
10000 & 00010 \\
00110 & 00001
\end{array}\right)
$$

Therefore, a quantum code [[5, 1, 2]] can be constructed from Eq. (44), where $d=2$, the Hamming weight of $\mathcal{G}_{5}$, can be calculated from the Hamming weight of the bitwise or of $g_{5}^{x}$ with $g_{5}^{z}$. It is the smallest code that can be not used for correcting any quantum errors, but for detecting only one quantum error in imperfect quantum channel.

Example 4.2 Considering the Pauli block matrix $\mathcal{Q}_{2}^{(2)}$ with the concatenated matrix $H_{2}=\left(H_{2}^{x} \mid H_{2}^{x}\right)$ in the field $G F(2)$, we select one row of $H_{2}$, denoted by $\mathcal{H}_{2}=$ $\left(h_{2}^{x} \mid h_{2}^{z}\right)=(10 \mid 01)$, from which we generate the stabilizer $\mathcal{S}$ with only one generator. Consequently, we obtain a generator matrix of the quantum code

$$
\mathcal{G}_{2}=\left(\begin{array}{l|l}
01 & 00 \\
00 & 10 \\
10 & 01
\end{array}\right)
$$

Therefore, we can construct a quantum code with the parameters [[2, 1, 1]]. It is known that this code can not correct or detect any quantum errors in the imperfect channel. However, based on the present 2-order Pauli block matrix $\mathcal{Q}_{2}$, one may obtain the combined $2^{m}$-order Pauli block matrices $\mathcal{Q}_{2}$, from which the stabilizer quantum codes $\left[\left[2^{m}, k, 2\right]\right]$ can be constructed with one-error-detecting capability [23].

Example 4.3 According to the Pauli block matrix $\mathcal{Q}_{3}^{(1)}$ with the concatenated matrix $H_{3}=\left(H_{3}^{x} \mid H_{3}^{x}\right)$ in the field $G F(3)$, we choose any two rows of $H_{3}$, say

$$
\mathcal{H}_{3}=\left(\begin{array}{l|l}
101 & 001 \\
011 & 100
\end{array}\right)
$$


from which we obtain the stabilizer $\mathcal{S}$ of a quantum code with two generators. Namely, we calculate the generator matrix of a quantum codes as

$$
\mathcal{G}_{3}=\left(\begin{array}{c|c}
011 & 000 \\
110 & 100 \\
000 & 010 \\
100 & 001
\end{array}\right) \text {. }
$$

It is obvious that the proposed quantum code has the parameters [[3, 1, 1]]. It also has no error-correction or error-detection capability. However, based on the combined Pauli block matrices $\mathcal{Q}_{3^{m}}$, we can always construct the quantum codes with the parameters $\left[\left[3^{m}, k, d \geq 2\right]\right]$.

Example 4.4 We consider the Pauli block matrix $\mathcal{Q}_{7}^{(1)}$ for $i=1$ and $j=3$ with the concatenated matrix expressed as

$$
H_{7}=\left(\begin{array}{l|l|l}
1110100 & 0001011 \\
0111010 & 1000101 \\
0011101 & 1100010 \\
1001110 & 0110001 \\
0100111 & 1011000 \\
1010011 & 0101100 \\
1101001 & 0010110
\end{array}\right) .
$$

For any six rows of $\mathrm{H}_{7}$, we may construct a quantum codes with the parameters $[[7,1,3]]$.

Example 4.5 We consider the Pauli block matrix $\mathcal{Q}_{6}$ with the concatenated matrix $H_{6}=\left(H_{6}^{x} \mid H_{6}^{x}\right)$. Selecting any five rows of $H_{6}$, say

$$
\mathcal{H}_{6}=\left(\begin{array}{l|l|l}
111100 & 100011 \\
111010 & 010101 \\
001111 & 110001 \\
100111 & 011100 \\
010111 & 101010
\end{array}\right),
$$

we obtain the generator matrix of a quantum code as

$$
\mathcal{G}_{6}=\left(\begin{array}{l|l|l}
111111 & 000000 \\
100010 & 100000 \\
010100 & 010000 \\
110000 & 001000 \\
011010 & 000100 \\
101100 & 000010 \\
001000 & 000001
\end{array}\right),
$$

from which a quantum code can be constructed with the parameters $[[6,1,2]]$. 
Example 4.6 Suppose $n=12$, and then we consider Pauli block matrix $\mathcal{Q}_{12}$ for $i=1$ and $j=3$ with the concatenated matrix $H_{12}$. It has been shown that all rows of $H_{12}$ are independent and commutative. Selecting any $12-k(0 \leq k \leq 12)$ row operators $\alpha_{i_{1}}, \alpha_{i_{2}}, \ldots, \alpha_{i_{12-k}}$ from $\mathcal{Q}_{12}^{(1)}$, we obtain the stabilizer $\mathcal{S}=\left\langle\alpha_{i_{1}}, \alpha_{i_{2}}, \ldots, \alpha_{i_{12-k}}\right\rangle$, from which quantum codes can be constructed with the parameters [[12,0,4]], [[12,1,4]], [[12,2,4]], [[12,3,3]], [[12,4,3]], [[12,5,3]], [[12,6,2]], [[12,7,2]], [[12,8,2]], [[12,9,2]], $[[12,10,2]]$, and [[12,11,2]].

\section{Conclusion}

A family of the stabilizer quantum codes is investigated with fast Pauli block transforms on the basis of quadratic residues in the finite field $G F(q)$. The present approach has the advantage of being fast encoding in terms of constructing the stabilizer. To gain the stabilizer of quantum codes, we investigate the construction approaches based on the designation of several Pauli block matrices with commutative row operators. The large-order Pauli block matrices can be structurally constructed via the fast Pauli block constructing (decomposing) transforms based on the recursive relationship of identity matrices and successively lower-order Pauli block matrices. These Pauli block matrices have such characteristics that all row operators are independent and commutative, which can be perfectly used for generating an Abelian operator group and hence for designing the stabilizer of our quantum codes. Furthermore, an instructive approach for smart constructions of quantum codes is suggested via the stabilizer formalism with respect to the yielded Abelian group $\mathcal{S}$ on the basis of the $n$-order Pauli block matrices $\mathcal{Q}_{n}$.

Acknowledgments This work has been supported by the National Natural Science Foundation of China (Grant No. 60902044), PhD Program Foundation of Ministry of Education of China (Grant No. 385 20090162120070), Postdoctoral Science Foundation of China (Grant No. 200801341), Science Foundation of Hunan Province (Grant No. 07JJ3128, 2008RS4016), and National Laboratory on Local Fiber-Optical Communication Network and Advanced Optical Communication System of Shanghai Jiaotong University, China.

Open Access This article is distributed under the terms of the Creative Commons Attribution Noncommercial License which permits any noncommercial use, distribution, and reproduction in any medium, provided the original author(s) and source are credited.

\section{References}

1. Gauss, C.F., Clarke, A.C.: Disquisitiones Arithemeticae. 2nd edn. Springer, New York (1986)

2. Jacobi, C.G.J.: Uber die Kreisteilung und ihre Anwendung auf die Zahlentheorie. Bericht Ak. Wiss, Berlin (1837)

3. Nielsen, M., Chuang, I.: Quantum Computation and Quantum Information. Cambridge University Press, Cambridge (2000)

4. Zeng, G., Guo, Y., Lee, M.H.: Stabilizer quantum codes over the Clifford algebra. J. Phys. A Math. Theor 41, 145304 (2008)

5. Knill, E., Laflamme, R.: A theory of quantum error-correcting codes. Phys. Rev. A 55, 900-911 (1997)

6. Calderbank, A.R., Rains, E.M., Shor, P.W., Sloane, N.J.A.: Quantum error correction and orthogonal geometry. Phys. Rev. Lett 78, 405-408 (1997) 
7. Calderbank, A.R., Shor, P.W.: Good quantum error-correction codes exist. Phys. Rev. A 54, 10981105 (1996)

8. Poulin, D.: Stabilizer formalism for operator quantum error correction. Phys. Rev. A 95, 230504 (2005)

9. Kribs, D., Laflamme, R., Poulin, D.: A unified and generalized approach to quantum error correction. Phys. Rev. Lett 94, 180501 (2005)

10. Aggarwal, V., Calderbank, A.R.: Boolean functions, projection operators, and quantum error correcting codes. IEEE. Trans. Inf. Theory 54(4), 1700-1708 (2008)

11. Steane, A.M.: Enlagement of Calderbank-Shor-Steane quantum codes. IEEE. Trans. Inf. Theory 45(7), 2492-2495 (1999)

12. Calderbank, A.R., Rains, E.M., Shor, P.W., Sloane, N.J.A.: Quantum error-correction via codes over GF(4). IEEE. Trans. Inf. Theory 44, 1369-1387 (1998)

13. MacKay, D.J.C., Mitchison, G.J., McFadden, P.L.: Sparse-graph codes for quantum error correction. IEEE. Trans. Inf. Theory 50, 2315-2330 (2004)

14. Matsumoto, R.: Improvement of Ashikhmin-Litsyn-Tsfasman bound for quantum codes. IEEE. Trans. Inf. Theory 48(7), 2122-2125 (2002)

15. Lee, M.H.: A new reverse jacket transform and its fast algorithm. IEEE Trans. Circuits Syst. II Analog Digit. Signal Process 47(1), 39-47 (2000)

16. Lee, M.H., Rajan, B.S., Park, J.Y.: A generalized reverse jacket transform. IEEE Trans. Circuits Syst. II Analog Digit. Signal Process 48(7), 684-691 (2001)

17. Lee, M.H., Zeng, G.: Family of fast jacket transform algorithms. Elect. Lett 43(11), 439-440 (2007)

18. Lee, M.H., Hou, J.: Fast block inverse jacket transform. IEEE Signal Process Lett. 13(8), 461464 (2006)

19. Zeng, G., Lee, M.H.: A generalized reverse block jacket transform. IEEE Trans. Circuits Syst. 55(6), 651-669 (2008)

20. Chen, Z., Lee, M.H., Zeng, G.: Fast cocyclic jacket transform. IEEE Trans. Signal Process 56(5), 21432148 (2008)

21. Paley, R.E.A.C.: On orthogonal matrices. J. Math. Phys. 12, 311-320 (1933)

22. Sugahara, M., Kruchinin, S.P.: Controlled not gate based on a two-layer system of the fractional quantum Hall effect. Mod. Phys. Lett. B 15(6), 473-477 (2001)

23. Guo, Y., Peng, J., Lee, M.H.: Fast quantum codes based on Pauli block jacket matrices. Quantum Inf. Process 8, 361-378 (2009) 